\title{
A Dutch Version of the Reiss Screen of Maladaptive Behavior
}

\author{
Agnes van Minnen, Petra M. Savelsberg, and Kees A. L. Hoogduin \\ Department of Clinical Psychology, University of Nijmegen
}

\begin{abstract}
The Reiss Screen for Maladaptive Behavior, an instrument used to identify mentally retarded persons with mental health problems, was evaluated with 89 mentally retanded adults. Reliability and validity of the Reiss Screen, as well as the relationship with subject characteristics, is reported for a Dutch population. Intemal consistency on the total score was good and modest on most subscales. The subscale autism had inadequate internal consistency. Interrater reliability was low to modest. Further, the Reiss Screen had good criterion validity. Last, few relationships were found between Reiss Screen scores and subject characteristics.
\end{abstract}

Prevalence rates of psychopathology in mentally retarded persons range from 14-17\% (Eaton \& Menolascino, 1982; Jacobson, 1982) to as high as 36-39\% (Iverson \& Fox, 1989; Reiss, 1990). This range in rates is in large part due to differences in applied methodology: In most cases different criteria to measure psychopathology have been used. Despite inconsistency in prevalence rates, it is assumed that mentally retarded persons have a higher chance of mental health problems than persons who are not mentally retarded (Jakab, 1982; Menolascino, Gilson, \& Levitas, 1986). Given this higher chance, it is essential to identify those persons who have mental health problems in addition to mental retardation, the so-called "dually diagnosed," because they are likely to be in need of mental health services. To aid in identifying the dually diagnosed, Reiss (1988) developed the Reiss Screen for Maladaptive Behavior.

Requests for reprints should be sent to Agnes van Minnen, Department of Clinical Psychology, University of Nijmegen, P.O. Box 9104, $6500 \mathrm{HE}$ Nijmegen, The Netherlands. 
The Reiss Screen is used to assess the probability that a mentally retarded adult has a significant mental health problem. The instrument is completed by informants who know the subject well. The Reiss Screen has 38 items, each representing a symptom of maladaptive behavior. The rating on each item ("no problem," "problem," or "major problem") is based on frequency, intensity, and consequences of the behavior. The Reiss Screen has eight subscales, each containing five items; "Aggressive Behavior," "Autism," "Psychosis," "Paranoia," "Depression-Behavioral Signs," "Depression-Physical Signs," "Dependent Personality Disorder," and "Avoidant Disorder." In addition to the subscales, six special maladaptive behavior items are included, each identifying a serious mental health problem. As a measure of the severity of mental health problems, a total score can be computed. Reiss used only 26 items to compute a total score; the sum of all item scores, excluding items of the autism scale items, the item "euphoria," and the six special maladaptive behavior items. The reason for not including all items in the total score was that at that time more normative data were available on the 26 -item score.

The purpose of this study was to evaluate the reliability, validity, and relationship with subject characteristics of the Reiss Screen in a Dutch population. Before the Dutch results are presented, results of previous research on the Reiss Screen are summarized.

Research on the Reiss Screen has been done with 621 mentally retarded persons in four separate subsamples. The results are described in the test manual (Reiss, 1988). Internal consistency was good for the 26-item score (alpha $=.84$ ), acceptable for most subscales, and low for the subscales depression-physical signs and autism (respectively, alpha $=.54$ and .57 ). The interrater reliability of items was poor (range $r=.30$ to $r=.79$, mean $r=.54$ ). The interrater reliability of the subscales ranged from .72 to .84 and was .75 for the 26 -item score. Due to the poor interrater reliability, Reiss (1988) recommended that the Reiss Screen be completed by two or more informants and that average ratings should be used.

Validity of the Reiss Screen was demonstrated in different ways. Significant relationships were found between Reiss Screen subscale scores and psychiatric diagnoses. Furthermore, persons with psychiatric diagnoses had significantly higher 26-item scores on the Reiss Screen than persons without a psychiatric diagnosis. These results are indicative of good criterion validity. High correlations were found between the CHEMRA, a predecessor test of the Reiss Screen and the Psychopathology Inventory of Mentally Retarded Adults (PIMRA) (Davidson, 1988, cited in Reiss, 1988). In a previous article, we reported a strong relationship between the Reiss Screen and the PIMRA (van Minnen, Savelsberg, \& Hoogduin, 1994). Miller and Monroe (1990) found high correlations between the Reiss Screen and the Adaptive Behavior Scales (ABS)-Part II, indicating good concurrent validity. 
Only a few significant relationships were found between Reiss Screen scores and subject characteristics. Women had significantly higher scores than men on the depression-behavioral signs scale. Subjects with a higher level of intelligence scored higher on the subscale aggression, and lower on the subscales depression-behavioral signs and paranoia, than subjects with a lower level of intelligence. No significant relationship with age was found.

In the following, the results of the Reiss Screen in a Dutch population are presented.

\section{METHOD}

\section{Subjects}

The subjects were 89 adults with a diagnosis of mild or borderline mental retardation drawn from two settings. Subjects in the first setting, the diagnosis group $(n=48)$, were treated as inpatients in a clinic for the treatment of mentally retarded adults with severe behavior disorders. All of these subjects had one or more psychiatric diagnosis, based on institutional records, in addition to mental retardation: personality disorder $(n=18)$, conduct disorder $(n=16)$, psychotic disorder $(n=10)$, affective disorder $(n=6)$, anxiety disorder $(n=5)$, adjustment disorder $(n=3)$, other $(n=3)$. Subjects in the second setting, the no-diagnosis group $(n=41)$, lived in residential facilities for mentally retarded adults, and none of them had been diagnosed with additional psychiatric disorders. Characteristics of the two samples in terms of age, sex, and level of intelligence are summarized in Table 1.

Members of the no-diagnosis group were significantly older $(t(87)$ $=2.51, p<.05)$ and had a significantly higher level of intelligence $(t(87)$ $=2.57, p<.05$ ) than members of the diagnosis group.

TABLE 1

Subject Characteristics

\begin{tabular}{llc}
\hline & $\begin{array}{c}\text { Diagnosis Group } \\
(n=48)\end{array}$ & $\begin{array}{c}\text { No-Diagnosis Group } \\
(n=41)\end{array}$ \\
\hline Age (years) & & \\
Mean & 35.1 & 41.2 \\
SD & 12.7 & 9.7 \\
Range & $19-67$ & $25-55$ \\
Sex (\%) & & \\
Male subjects & 72.9 & 63.4 \\
$\quad$ Female subjects & 27.1 & 36.6 \\
Level of intelligence & & \\
Mean & 72.0 & 77.4 \\
SD & 10.2 & 9.5 \\
Range & $54-100$ & $52-90$ \\
\hline
\end{tabular}




\section{Procedure}

The Reiss Screen was translated into Dutch by a translation-retranslation method. For each subject, the Reiss Screen was completed by two independent informants on the same day. Both informants were ward staff members who had known the subject at least 3 months, the first informant being the subject's primary contact person.

\section{Data Analysis}

The data were analyzed using the SPSS-X package (SPSS, 1990). The first informants' ratings were used for the analyses of the Reiss Screen. In this study, in addition to the 26-item score used by Reiss (1988), a total score was calculated using the sum of all 38 items.

\section{RESULTS}

\section{Reliability}

Internal consistency. The coefficient alpha was .92, and the Spearman Brown split-half coefficient was .91 for both the total score and the 26-item score, indicating a high level of internal consistency. Alpha coefficients of the subscales ranged from .46 to .87 . All scales, except for the autism scale, had an acceptable level of internal consistency. Alpha coefficients for the scales are shown in Table 2.

Interrater reliability. Correlation coefficients (Pearson $r$ ) were computed for the two independent informants' Reiss Screen scores to assess interrater

TABLE 2

Reliability: Alpha Coefincients of the Reiss Screen Scales and Correlations (Pearson $r$ ) Between the two Informants' Scores $(n=89)$

\begin{tabular}{lccc}
\hline Scale & $n$ of Items & Alpha & Pearson $r^{*}$ \\
\hline Aggressive behavior & 5 & 0.87 & .68 \\
Autism & 5 & 0.46 & .71 \\
Psychosis & 5 & 0.68 & .79 \\
Paranoia & 5 & 0.72 & .67 \\
Depression (B) & 5 & 0.83 & .64 \\
Depression (P) & 5 & 0.76 & .84 \\
Dependent PD & 5 & 0.69 & .70 \\
Avoidant disorder & 5 & 0.71 & .50 \\
Total score & 38 & 0.92 & .81 \\
26-item score & 26 & 0.92 & .80 \\
\hline
\end{tabular}

*Significance at $p<.001$ 
reliability and are shown in Table 2 . The correlation between the two ratings on the subscales ranged from .50 to .84 . The correlation between the two informants on the total score was .81 , and .80 on the 26-item score. All correlations were significant at $p<.001$. Furthermore, differences in mean scales' scores were tested (paired $t$-test) between the two informants. Although the first informants scored higher on most scales than the second informants, this was only significant for the subscale depression-behavioral signs $(t(88)=2.0, p<.05)$.

\section{Validity}

Table 3 gives the diagnosis and no-diagnosis groups' mean scores and standard deviations on Reiss Screen scales. Differences between means were computed by means of two-tailed $t$-tests. Subjects with diagnosed psychopathology had significantly higher total Reiss Screen scores $(t(87)$ $=7.20, p<.001)$ and 26 -item scores $(t(87)=6.86, p<.001)$ than those with no psychiatric diagnosis. All Reiss Screen subscales could significantly distinguish between persons with and without diagnosed psychopathology $(p<.001)$. Although the scales were not validated to the diagnosis the persons had in their records, these findings are evidence for a good criterion validity.

\section{Relationship Between Subject Characteristics and Reiss Screen Scores}

The variables age and level of intelligence were found not to be significantly correlated to Reiss Screen scores. Women in both groups scored

TABLE 3

Mean Scores and Standard Deviations on Reiss Screen Scales of the Diagnosis Group and the No-Diagnosis Group

\begin{tabular}{lccccc}
\hline & \multicolumn{2}{c}{$\begin{array}{c}\text { Diagnosis } \\
(n=48)\end{array}$} & & \multicolumn{2}{c}{$\begin{array}{c}\text { No-Diagnosis } \\
(n=41)\end{array}$} \\
\cline { 6 - 7 } & Mean & $S D$ & & Mean* & $S D$ \\
Scale & 3.8 & 2.8 & & 0.5 & 1.0 \\
Aggressive behavior & 1.6 & 1.5 & & 0.3 & 0.8 \\
Autism & 2.4 & 2.1 & & 0.8 & 1.1 \\
Psychosis & 3.1 & 2.3 & & 0.9 & 1.3 \\
Paranoia & 3.1 & 2.7 & & 0.7 & 1.0 \\
Depression (B) & 2.0 & 2.4 & 0.7 & 1.0 \\
Depression (P) & 3.3 & 2.3 & 1.5 & 1.4 \\
Dependent PD & 3.0 & 2.3 & 0.9 & 1.1 \\
Avoidant disorder & 17.7 & 11.0 & 4.6 & 4.5 \\
Total score & 15.0 & 9.7 & 4.0 & 3.6 \\
26-item score & & & &
\end{tabular}

*Significance at $p<.001$. 
significantly higher than men on the depression-physical signs subscale $(t(87)=2.97, p<.05)$. Other significant relations between sex and Reiss Screen scores were not found.

\section{DISCUSSION}

In this study a total score consisting of all 38 items of the Reiss Screen was used. The results regarding reliability and validity of this total score did not differ fundamentally from findings from this or previous studies in which a 26-item score was used. Because the total score refers to an indication of severity of mental health problems and to the identification of a dual diagnosis, it seems preferable to include scores of all 38 items in the total score, instead of only 26 items. The Reiss Screen total scale showed good internal consistency. Internal consistency of most subscales was acceptable to good. Consistent with previous research, the subscale autism had inadequate internal consistency. In this study, this may be due to the fact that persons diagnosed with autism were not equally represented in the research population.

Interrater reliability of the Reiss Screen was low to modest. This may be due to differences in the informants' perceptions of the subject. To take average scores of different informants, as suggested by Reiss, is one way to solve this problem. Low interrater reliability may, however, also be due to the Reiss Screen' multiple-criteria rating-system. Therefore, it may be worthwhile to make the criteria of the rating system more concrete.

On the total score, as well as on all subscale scores, it was possible to distinguish persons with diagnosed psychopathology from persons without psychopathology. This is an indication that the Reiss Screen indeed is a worthwhile aid in the identification of the dually diagnosed. The only relationship between subject characteristics and Reiss Screen scores was that women scored significantly higher than men on the depression-physical signs scale. This is consistent with the findings of the study of Reiss, in which women scored higher than men on the depression-behavioral signs scale. Other subject characteristics didn't seem to be related to the Reiss Screen scores.

In conclusion, the findings of the Dutch study are highly consistent with results of studies using the English version of the Reiss Screen. This provides support for the reliability and validity of the Reiss Screen in assessing mental health problems of persons with mental retardation across a cultural setting. The findings of this study show that the Reiss Screen is most reliable and valid when using the total score. Although the reliabilities for the total score are adequate for individual decision making, the subscales' levels of reliability indicate that the use of the subscales is limited to research purposes. The Reiss Screen can be useful as a screening measure- 
ment to identify persons with dual diagnosis. For persons who have a high probability of mental health problems as indicated by the Reiss Screen, other assessment methods should be used for more specific information and individual decision making. Given the fact that not all diagnoses were represented in the research sample, in this study it was not possible to validate diagnoses with the specific scales of the Reiss Screen. In future research it would be worthwhile to study whether the different scales comprising the Reiss Screen can be used to identify persons with particular mental health problems.

\section{REFERENCES}

Davidson, M. (1988). Psychometric Characteristics of the Checklist of Emotional Problems with Mentally Retarded Adults. Cited in S. Reiss. The Reiss Screen Test manual (p. 62). Orland Park, IL: International Diagnostic Systems, Inc.

Eaton, L. F., \& Menolascino, F. J. (1982). Psychiatric disorders in the mentally retarded: Types, problems and challenges. American Journal of Psychiatry, 139, 1297-1303.

Iverson, J. C., \& Fox, R. A. (1989). Prevalence of psychopathology among mentally retarded adults. Research in Development Disabilities, 10, 77-83.

Jacobson, J. W. (1982). Problem behavior and psychiatric impairment within a developmentally disabled population: I. Behavior frequency. Applied Research in Mental Retardation, 3, 121-139.

Jakab, I. (1982). Psychiatric disorders in mental retardation: Recognition, diagnosis and treatment. In I. Jakab (Ed.), Mental retardation (pp. 270-326). New York: Karger.

Menolascino, F. J., Gilson, S. F., \& Levitas, A. S. (1986). Clinical care update: Issues in the treatment of mentally retarded patients in the community mental health system. Community Mental Health Journal, 22, 314-327.

Miller, L. K., \& Monroe, M. J.(1990). Musical aptitude and adaptive behavior of people with mental retardation. American Journal on Mental Retardation, 95, 220-227.

Reiss, S. (1988). The Reiss Screen Test manual. Orland Park, IL: International Diagnostic Systems, Inc.

Reiss, S. (1990). Prevalence of dual diagnosis in community-based day programs in the Chicago metropolitan area. American Journal on Mental Retardation, 94, 578-585.

SPSS. (1990.) SPSS reference guide. Chicago: SPSS Inc.

van Minnen, A., Savelsberg, P. M., \& Hoogduin, C. A. L. (1994). A Dutch version of the Psychopathology Inventory for Mentally Retarded Adults (PIMRA): Psychometric properties, correlation between self-report and rating-by-others forms, relationship with behavior problems and subject characteristics. Research in Developmental Disabilities, 15, 269-278. 